\title{
Moving towards a better understanding of potential pitfalls in quantitative PET myocardial blood flow
}

\author{
Paul C. Cremer, MD, ${ }^{a}$ Frank P. DiFilippo, PhD, ${ }^{b}$ and Wael A. Jaber, MD $^{\mathrm{a}}$ \\ a Heart and Vascular Institute, Cleveland Clinic, Cleveland, $\mathrm{OH}$ \\ b Imaging Institute, Cleveland Clinic, Cleveland, $\mathrm{OH}$
}

Received Jan 20, 2017; accepted Jan 23, 2017

doi: 10.1007/s12350-017-0828-9

\section{See related article, pp. 1286-1295}

Never mistake motion for action.

- Ernest Hemingway

Rest-stress myocardial perfusion position emission tomography (PET) is a well-established non-invasive imaging test to evaluate patients with known or suspected coronary artery disease (CAD). In certain clinical scenarios, especially in patients that cannot exercise, rest-stress myocardial perfusion PET may be a preferred, first-line test. ${ }^{1}$ In part, this recommendation is based on robust, albeit predominantly retrospective, data from large cardiac PET centers. ${ }^{2}$ Even with only relative perfusion data, myocardial perfusion PET may provide superior diagnostic accuracy and reader confidence compared to single-photon emission computed tomography (SPECT). ${ }^{3}$ Moreover, dynamic cardiac PET allows quantification of myocardial blood flow (MBF), which can improve cardiovascular risk stratification ${ }^{4}$ and help to exclude high-risk coronary artery disease. ${ }^{5}$

Given these advantages, more widespread clinical use of rest-stress myocardial perfusion PET with quantitative MBF seems justified. However, like any medical innovation, technical issues and practical considerations become more apparent as the technology is disseminated. In particular, issues related to reproducibility are of paramount importance, and in general, reproducibility may be impaired either due to differences in data acquisition or data analysis. Regarding different

Reprint requests: Wael A. Jaber, Heart and Vascular Institute, Cleveland Clinic, Cleveland, OH 44195; jaberw@ccf.org.

J Nucl Cardiol 2018;25:1296-8.

$1071-3581 / \$ 34.00$

Copyright (C) 2017 American Society of Nuclear Cardiology. analyses in the same patients, the relationship between cardiac mortality and stress MBF is highly variable depending on the input function and extraction model employed, though the relationship between cardiac mortality and coronary flow reserve (CFR) is more consistent. ${ }^{6}$ Similarly, when point spread function modeling and time-of-flight reconstructions are compared to standard PET reconstructions, CFR results are consistent, but marked variations in rest and stress MBF have been reported. ${ }^{7}$ Fortunately, when the same data set is analyzed with different automated software programs, MBF results are comparable, provided the analysis is performed with the same one-compartment model of ${ }^{82} \mathrm{Rb}$ kinetics. ${ }^{8}$ Regarding variations in data acquisition, the vasodilator and timing of radionuclide injection both influence maximal hyperemia. ${ }^{9}$ Moreover, the method of radionuclide infusion is also important. Recently, a constant activity rate infusion has been shown to improve reproducibility when compared to administration at a constant flow rate. ${ }^{10}$

In addition to these concerns, patient motion can also dramatically impact data acquisition and the fidelity of quantitative PET MBF. ${ }^{11,12}$ In contrast to SPECT, where cine raw projection images are available for routine evaluation of patient motion, a conventional clinical PET protocol (static and gated imaging) does not provide the clinician a comparable cine image from which to evaluate patient motion. However, dynamic PET is "4D imaging" with a series of tomographic frames, and inter-frame motion of the heart is visible. Consequently, MBF software vendors are now including motion correction algorithms to improve the accuracy of MBF calculations. With PET, patient motion not only causes image artifacts but also affects the accuracy of attenuation correction. Prior to applying CT-based attenuation correction, typical clinical software allows the technologist to correct for misregistration for single time-frame images (static and gated) to a certain extent. Temporal misregistration from patient motion remains, however, and clinical tools for routine registration of 
dynamic PET data with CT images for the purpose of PET 4D attenuation correction are greatly needed.

In the current issue, Memmott and colleagues investigate a novel aspect of data acquisition that could impact the accuracy and reproducibility of MBF quantification-the interaction between specific protocols, vasodilator, and patient motion. ${ }^{13}$ The authors performed a retrospective cohort study with 30 consecutive patients that had dynamic ${ }^{82} \mathrm{Rb}$ PET/CT myocardial perfusion compared to two similar cohorts, one with stress via adenosine and the other with stress via an incremental adenosine protocol. Their outcome was the severity of patient motion, assessed via a qualitative scale and with quantitative frame-to-frame pixel shifts. Per local protocols, regadenoson was used only in patients $>140 \mathrm{~kg}$ or in patients with controlled asthma or COPD. Regadenoson $(400 \mu \mathrm{g})$ was injected over 20 seconds and imaging was performed 40 seconds after injection. Patients with respiratory disease could also receive an incremental adenosine protocol that included an infusion at $50 \mu \mathrm{g} / \mathrm{kg} / \mathrm{min}$ for 1 minute, $100 \mu \mathrm{g} / \mathrm{kg} / \mathrm{min}$ for 1 minute, and $140 \mu \mathrm{g} / \mathrm{kg} / \mathrm{min}$ for 4 minutes with imaging at 4 minutes into the infusion. In patients without lung disease or obesity, adenosine was infused at $140 \mu \mathrm{g} / \mathrm{kg} / \mathrm{min}$ for 4.5 minutes, and imaging was started 2 minutes into infusion. For all patients, list mode data acquisition was performed for 7 minutes.

First, in a separate cohort of approximately 3000 patients, Memmott and colleagues show that many more patients had considerable motion with adenosine stress versus rest. In this analysis, patients serve as their own internal control, and the results argue that the vasodilator is associated with motion. Next, the authors show a statistically detectable difference in both the quantitative and qualitative motion scores for patients with either adenosine or incremental adenosine compared to patients that receive regadenoson. Interestingly, most of the motion was non-returning and occurred later in the study, prompting the authors to suggest that termination of the adenosine infusion may have been responsible.

Overall, the strengths of this study are its focused hypothesis, distinct protocols, and meticulous attention to technical details. However, the implications of any observational study are also dependent on a carefully selected control group. For the current study, a more ideal design would have been a blinded crossover study where patients serve as internal controls, undergoing separate stress acquisitions with regadenoson and adenosine. Given practical considerations, this design may not have been feasible. Nonetheless, Memmott and colleagues attempted to control for bias only by weight and respiratory status, and other variables, such as patient age, may also contribute to patient motion. In addition, by design, the current study did not assess the impact of motion on the sensitivity and specificity of perfusion findings or quantitative MBF analysis.

Similar to most interesting investigations, the current study raises more questions than answers. First, shorter adenosine infusion times may be reasonable in SPECT,${ }^{13}$ but given the association between adenosine termination and motion, would a standard 6-min infusion lead to less motion? Second, can motion correction enhance reproducibility and interpretability? Such an investigation would need to be conducted in a blinded fashion before and after motion correction. Finally, and most importantly, the current investigation on patient motion highlights the need for action from ASNC and vendors to standardize protocols related to the acquisition and analysis of PET myocardial perfusion data so that the advantages of quantitative PET MBF can be more fully realized.

\section{References}

1. Bateman TM, Dilsizian V, Beanlands RS, DePuey EG, Heller GV, Wolinsky DA. ASNC/SNMMI Joint Position Statement on the Clinical Indications for Myocardial Perfusion PET. J Nucl Cardiol. 2016. doi:10.1007/s12350-016-0626-9.

2. Gould KL, Johnson NP, Bateman TM, Beanlands RS, Bengel FM, Bober R, et al. Anatomic versus physiologic assessment of coronary artery disease: role of coronary flow reserve, fractional flow reserve, and positron emission tomography imaging in revascularization decision-making. J Am Coll Cardiol 2013;62:1639-53.

3. McArdle BA, Dowsley TF, deKemp RA, GA Wells, Beanlands RS. Does Rubidium-82 PET have superior accuracy to SPECT perfusion imaging for the diagnosis of obstructive coronary disease? A systematic review and meta-analysis. J Am Coll Cardiol 2012;60:1828-37

4. Murthy VL, Naya M, Foster CR, Hainer J, Gaber M, Di Carli G, et al. Improved cardiac risk assessment with noninvasive measures of coronary flow reserve. Circulation 2011;124:2215-24.

5. Naya M, Murthy VL, Taqueti VR, Foster CR, Klein J, Garber M, et al. Preserved coronary flow reserve effectively excludes highrisk coronary artery disease on angiography. J Nucl Med 2014;55:248-55.

6. Murthy VL, Lee BC, Sitek A, Naya M, Moody J, Polavarapu V, et al. Comparison of prognostic validation of multiple methods of quantification of myocardial blood flow with ${ }^{82} \mathrm{Rb}$ PET. J Nucl Med 2014;55:1952-8.

7. deKemp RA, Declerk J, Klein R, Pan XB, Nakazato R, Tonge C, et al. Multisoftware reproducibility study of stress and rest myocardial blood flow assessed with 3D dynamic PET/CT and a 1-tissue-compartment model of ${ }^{82} \mathrm{Rb}$ kinetics. J Nucl Med 2013;54:571-7.

8. Armstrong IS, Tonge CM, Arumugam P. Impact of point spread function modeling and time-of-flight on myocardial blood flow and myocardial flow reserve measurements for rubidium- 82 cardiac PET. J Nucl Cardiol 2014;21:467-74.

9. Johnson NP, Gould KL. Regadenoson versus dipyridamole hyperemia for cardiac PET imaging. J Am Coll Cardiol Imaging 2015;8:438-47.

10. Klein R, Ocneanu A, Renaud JM, Ziadi MC, Beanlands RS. Consistent tracer administration profile improves test-retest repeatability of myocardial blood flow quantification with ${ }^{82} \mathrm{Rb}$ 
dynamic PET imaging. J Nucl Cardiol. 2016. doi: 10.1007/s12350-016-0698-6.

11. Koshino K, Watabe H, Enmi J, Hirano Y, Zeniya T, Hasegawa S, et al. Effects of patient movement on measurements of myocardial blood flow and viability in resting ${ }^{15} \mathrm{O}$-water PET studies. J Nucl Cardiol 2012;19:524-33.
12. Rajaram M, Tahari AK, Lee AH, Lodge MA, Tsui B, Nekolla S, et al. Cardiac PET/CT misregistration causes significant changes in estimated myocardial blood flow. J Nucl Med 2013;54:50-4.

13. Henzlova MJ, Duvall WL, Einstein AJ, Travin MI, Verberne HJ. ASNC imaging guidelines for SPECT nuclear cardiology procedures: Stress, protocols, and tracers. doi:10.1007/s12350-015-0387-x. 